\title{
Performance analysis of distributed control configurations in LQR multi-agent system design
}

\author{
Ivana Tomić \\ School of Mathematics, Computer Science \\ and Engineering, City University, \\ Northampton Square, London EC1V 0HB, UK \\ Email: Ivana.Tomic.1@city.ac.uk
}

\author{
George D. Halikias \\ School of Mathematics, Computer Science \\ and Engineering, City University, \\ Northampton Square, London EC1V OHB, UK \\ Email: G.Halikias@city.ac.uk
}

\begin{abstract}
The paper considers a distributed Linear Quadratic Regulator (LQR) design framework for a network of identical dynamically decoupled multi-agent systems. It is known that in this case a stabilizing distributed controller for the network can be obtained by solving a centralized LQR problem whose size depends on the maximum vertex degree of the graph. A systematic method is presented for computing the performance loss of various distributed control configurations relative to the performance of the centralized controller. A procedure is developed for analyzing the performance loss for general distributed control configurations and state-space directions. It is also shown that by removing a single link we can always define a control configuration for which there is no performance loss, provided the initial state of the aggregate system lies in a particular direction of state-space which is identified. The results are illustrated by an exhaustive analysis of the network consisting of six identical agents.
\end{abstract}

\section{INTRODUCTION}

Recently control of multi-agent systems has received considerable attention due to its broad spectrum of applications, e.g. formation control [1]-[3], satellite clustering [4], flocking [5]-[6], distributed sensor networks [7], air traffic control [8], congestion control in communication networks [9], etc.

There are three possible control methods of multi-agent systems, centralized, decentralized and distributed control. Optimal performance is achieved by a centralized controller, however this structure becomes infeasible as the number of subsystems and the distance between them increases. Comparison of all these methods has been undertaken in [10]. Conclusions tend to favour distributed control which involves local information exchange between subsystems. Often the information exchange is captured as a graph, and many researchers have obtained novel results by combining graph theory and control approaches, see e.g. [11]-[13].

The analysis of a formation of interacting and cooperating identical subsystems was first proposed in [2]; in this work the communication topology of the network was modeled using graph theory and necessary and sufficient stability conditions were derived. This framework was used in [14] to establish robust controller properties for an arbitrary communication topology, whereas previous solutions were adequate only for undirected communication networks.

Linear Quadratic Regulator (LQR) theory has been successfully applied to the control of multi-agent systems due to its guaranteed robustness properties. For example, in [15] an LQR-based method was proposed for optimal control of multi-vehicle systems with single-integrator dynamics in a continuous-time setting. In [16], the authors analyzed the influence of the topology of the interconnection graph on the closed-loop performance achieved by subsystems in a distributed LQR framework. In [17], the authors proposed a Linear Matrix Inequality (LMI) based distributed LQR design with guaranteed LQR cost for identical dynamically coupled systems where the solution depends on the total number of agents. An estimate of the bound on the maximum time delay that can be accommodated was also obtained. In [18], it was shown that the distributed LQR control law guarantees not only optimization performance at the network level but also a convergence rate for the group of subsystems. An alternative approach to structured distributed controllers that has appeared in literature is given in [19]-[20]. By employing the augmented Lagrangian method the structured optimal feedback gains can be designed without the knowledge of a stabilizing structured gain to initiate the algorithm.

The present work is based on the framework defined in [21] where a distributed LQR design strategy for dynamically decoupled multi-agent systems has been introduced. The subsystems can be actuated independently, but share a common objective which forces them to interact with each other. Coupling between subsystems is described by a communication graph, at each node of which the models of the neighbouring nodes are used to predict its behaviour. Such an approach leads to an elegant and powerful result: a stabilizing distributed controller can be found by solving a single LQR problem whose size depends on the maximum vertex degree of the graph.

The aim of the present paper is to compare the optimal centralized LQR controller with the family of distributed suboptimal controllers presented in [21] by computing the loss of performance introduced by the distributed law. Using perturbation analysis it is shown that we can always choose a distributed-control configuration by removing a single link, along with a direction in state-space of the aggregate system for which there is no performance loss. A procedure is also developed to analyze the performance loss for general control configurations and state-space directions. 
The remainder of this paper is organized as follows. Section II contains preliminary notation, including a brief summary of relevant results from graph theory. Section III presents the centralized controller design procedure. The proposed design is extended to the distributed case using local LQR solution properties. Section IV presents the main results of the paper. Distributed control configurations and initial statespace directions are identified for which no performance loss occurs relative to the LQR optimal centralized controller. A procedure is also presented for analyzing the performance cost of an arbitrary distributed configuration. Presented results are illustrated by an exhaustive analysis of of the network consisting of six identical agents. Finally, the paper's conclusions appear in Section V.

\section{Preliminaries}

\section{A. Notation and Definitions}

The following notation will be used throughout the paper: $I_{n}$ denotes the identity matrix of dimension $n, I_{n} \in \mathbb{R}^{n \times n} ; M^{T}$ and $\boldsymbol{a}^{T}$ denote the transpose of matrix $M$ and the transpose of column vector $\boldsymbol{a}=\left[a_{1}, \ldots, a_{n}\right]^{T}$, respectively; $A \otimes B$ denotes the Kronecker product of $A$ and $B$. Let $A \in \mathbb{R}^{m \times n}$ and $B \in \mathbb{R}^{p \times q}$, then:

$$
A \otimes B=\left(\begin{array}{cccc}
a_{11} B & a_{12} B & \ldots & a_{1 n} B \\
\vdots & \vdots & \ddots & \vdots \\
a_{m 1} B & a_{m 2} B & \ldots & a_{m n} B
\end{array}\right) \in \mathbb{R}^{m p \times n q}
$$

The spectrum of $n \times n$ matrix $M$ is denoted as $\mathcal{S}(M)=$ $\left\{\lambda_{1}(M), \lambda_{2}(M), \ldots, \lambda_{n}(M)\right\}$. If the spectrum is real $\lambda_{i}(M)$ denotes the $i$ th eigenvalue of $M$ indexed in decreasing order.

Definition 1. A matrix $M \in \mathbb{R}^{n \times n}$ is called stable or Hurwitz if all its eigenvalues have negative real part, i.e. $\mathcal{S}(M) \subseteq \mathbb{C}_{\text {_. }}$.

\section{B. Graph Theory Preliminaries}

The underlying network is represented as a graph, described by $\mathcal{G}=(\mathcal{V}, \mathcal{E})$, where $\mathcal{V}$ is the set of nodes (or vertices), $\mathcal{V}=\{1,2, \ldots, N\}$, and $\mathcal{E} \subseteq \mathcal{V} \times \mathcal{V}$ is the set of edges, $\mathcal{E} \subseteq$ $\{(i, j): i, j \in \mathcal{V}, j \neq i\}$. If $i, j \in \mathcal{V}$ and $(i, j) \in \mathcal{E}$, then $i$ and $j$ are said to be adjacent (or neighbors) which is denoted as $i \sim j$. We assume that there is no edge from a node to itself (i.e. no self loops) and that the edge between nodes $i$ and $j$ is undirected. For an undirected graph the communication between two nodes (or agents) is bidirectional. An undirected graph is said to be complete if every pair of distinct nodes is connected by a unique edge. The number of neighbors of each node, $d_{i}$ for $i=1,2, \ldots, N$, is called its degree or valency. Let $d_{\max }(\mathcal{G})$ and $d_{\min }(\mathcal{G})$ denote the maximum and minimum node degree of the graph $\mathcal{G}$, respectively. Any undirected graph can be represented by its adjacency matrix, $\mathbf{A}(\mathcal{G})$. Let $\mathbf{A}_{i, j} \in$ $\mathbb{R}$ be the $(i, j)$ element of $\mathbf{A}(\mathcal{G})$, then the following is true: $\mathbf{A}_{i, i}=0, \forall i=1,2, \ldots, N$ and

$$
\mathbf{A}_{i, j}=\left\{\begin{array}{l}
0 \text { if }(i, j) \notin \mathcal{E} \forall i, j=1,2, \ldots, N, \quad i \neq j, \\
1 \text { if }(i, j) \in \mathcal{E} \forall i, j=1,2, \ldots, N, \quad i \neq j .
\end{array}\right.
$$

\section{PROBlem FORMULATION}

\section{A. Centralized LQR Controller for Dynamically Decoupled} Systems

Consider $i$ th dynamical system whose dynamics can be described by the continuous-time state equation:

$$
\dot{\boldsymbol{x}}_{\boldsymbol{i}}(t)=A \boldsymbol{x}_{\boldsymbol{i}}+B \boldsymbol{u}_{\boldsymbol{i}}, \quad \boldsymbol{x}_{\boldsymbol{i}}(0)=\boldsymbol{x}_{\boldsymbol{i} \mathbf{0}}
$$

where $A \in \mathbb{R}^{n \times n}, B \in \mathbb{R}^{n \times m}$ and $\boldsymbol{x}_{\boldsymbol{i}}(t) \in \mathbb{R}^{n}, \boldsymbol{u}_{\boldsymbol{i}}(t) \in \mathbb{R}^{m}$ are the state and input vectors of the $i$ th system at time $t$, respectively. Then, the dynamics of these $N$ subsystems, indexed as $1,2, \ldots, N$, considered in total, are described by

$$
\dot{\boldsymbol{x}}(t)=A_{a} \boldsymbol{x}+B_{a} \boldsymbol{u}, \boldsymbol{x}(0)=\boldsymbol{x}_{\mathbf{0}}
$$

where the column vectors $\boldsymbol{x}(t)=\left[\boldsymbol{x}_{\mathbf{1}}^{T}(t), \ldots, \boldsymbol{x}_{\boldsymbol{N}}^{T}(t)\right]^{T}$ and $\boldsymbol{u}(t)=\left[\boldsymbol{u}_{\mathbf{1}}^{T}(t), \ldots, \boldsymbol{u}_{\boldsymbol{N}}^{T}(t)\right]^{T}$ collect the states and inputs of the $N$ systems, while $A_{a}=I_{N} \otimes A$ and $B_{a}=I_{N} \otimes B$, with $A$ and $B$ defined as in (1).

The LQR problem for the system (2) is described through the cost function which contains terms for weighting the difference between $i$ th and $j$ th system states, as well as the $i$ th system state and input:

$$
\begin{array}{r}
J\left(\boldsymbol{u}(t), \boldsymbol{x}_{\mathbf{0}}\right)=\int_{0}^{\infty}\left(\sum_{i=1}^{N}\left(\boldsymbol{x}_{\boldsymbol{i}}(t)^{T} Q_{i i} \boldsymbol{x}_{\boldsymbol{i}}(t)+\boldsymbol{u}_{\boldsymbol{i}}(t)^{T} R_{i i} \boldsymbol{u}_{\boldsymbol{i}}(t)\right)+\right. \\
\left.\sum_{i=1}^{N} \sum_{\substack{j=1 \\
j>i}}^{N}\left(\left(\boldsymbol{x}_{\boldsymbol{i}}(t)-\boldsymbol{x}_{\boldsymbol{j}}(t)\right)^{T} Q_{i j}\left(\boldsymbol{x}_{\boldsymbol{i}}(t)-\boldsymbol{x}_{\boldsymbol{j}}(t)\right)\right)\right) \mathrm{d} t .
\end{array}
$$

which can be rewritten using the more compact notation:

$$
J\left(\boldsymbol{u}(t), \boldsymbol{x}_{\mathbf{0}}\right)=\int_{0}^{\infty}\left(\boldsymbol{x}(t)^{T} Q_{a} \boldsymbol{x}(t)+\boldsymbol{u}(t)^{T} R_{a} \boldsymbol{u}(t)\right) \mathrm{d} t
$$

where the matrices $Q_{a}$ and $R_{a}$ have the following structure:

$$
Q_{a}=\left(\begin{array}{cccc}
Q_{a_{11}} & Q_{a_{12}} & \ldots & Q_{a_{1 N}} \\
Q_{a_{21}} & Q_{a_{22}} & \ldots & Q_{a_{2 N}} \\
\vdots & \vdots & \ddots & \vdots \\
Q_{a_{N 1}} & Q_{a_{N 2}} & \ldots & Q_{a_{N N}}
\end{array}\right), R_{a}=I_{N} \otimes R
$$

with $Q_{a_{i i}}=\sum_{k=1}^{N} Q_{i k}$ for $i=1, \ldots, N, Q_{a_{i j}}=-Q_{i j}$ for $i, j=1, \ldots, N, i \neq j$, and $R_{i i}=R_{i i}^{T}>0, \forall i$. Further, $Q_{i i}=Q_{i i}^{T} \geq 0, \forall i$ and $Q_{i j}=Q_{i j}^{T}=Q_{j i} \geq 0 \quad \forall i \neq j$.

The following assumptions apply throughout this section for $Q=Q^{T} \geq 0$ and $Q_{a}$ as in (4).

Assumption 1. The pair $(A, B)$ is stabilizable and the pair $(A, C)$ is observable, where $C$ is any matrix such that $C^{T} C=$ $Q$.

Assumption 2. The pair $\left(A_{a}, B_{a}\right)$ is stabilizable and the pair $\left(A_{a}, C_{a}\right)$ is observable, where $C_{a}$ is any matrix such that $C_{a}^{T} C_{a}=Q_{a}$.

Correspondingly, given the initial conditions, $x_{\mathbf{0}}$, the control input $\boldsymbol{u}=-R_{a}^{-1} B_{a}^{T} P_{a} \boldsymbol{x}$ minimizes the cost function in (3) subject to $\dot{\boldsymbol{x}}(t)=A_{a} \boldsymbol{x}+B_{a} \boldsymbol{u}, \boldsymbol{x}(0)=\boldsymbol{x}_{\mathbf{0}}$, where $P_{a}$ is the symmetric positive definite stabilizing solution of the following (large-scale) Algebraic Riccati Equation (ARE):

$$
A_{a}^{T} P_{a}+P_{a} A_{a}-P_{a} B_{a} R_{a}^{-1} B_{a}^{T} P_{a}+Q_{a}=0 .
$$


If the weighting matrices of the (large-scale) LQR problem (2)-(3) are chosen as $Q_{a_{i i}}=Q_{1} \forall i=1, \ldots, N$, and $Q_{a_{i j}}=$ $Q_{2} \forall i=1, \ldots, N, i \neq j$ and $P_{a} \in \mathbb{R}^{n N \times n N}$ is the stabilizing solution of (5) whose individual blocks are denoted as $P_{a_{i j}}=$ $P_{a}[(i-1) n+1: i n,(j-1) n+1: j n]$ with $i, j=1, \ldots, N$. Then, the following are true:

The unique symmetric positive definite solution to (5) has the structure:

$$
P_{a}=\left(\begin{array}{cccc}
P_{a_{11}} & P_{a_{12}} & \ldots & P_{a_{12}} \\
P_{a_{12}} & P_{a_{11}} & \ldots & P_{a_{12}} \\
\vdots & \vdots & \ddots & \vdots \\
P_{a_{12}} & \ldots & \ldots & P_{a_{11}}
\end{array}\right)
$$

in which the diagonal blocks can be expressed as: $P_{a_{11}}=$ $P-(N-1) P_{a_{12}} . P \in \mathbb{R}^{n \times n}$ is the symmetric positive definite solution of the ARE:

$$
A^{T} P+P A-P B R^{-1} B^{T} P+Q_{1}=0 .
$$

All off-diagonal blocks of $P_{a}$, namely $P_{a_{i j}}$ for $i \neq j$, are equal symmetric negative semi-definite matrices, denoted as $P_{a_{12}} \leq 0$. Further, the (large-scale) LQR gain matrix $K_{a}$ is of the form:

$$
K_{a}=\left(\begin{array}{cccc}
K_{a_{11}} & K_{a_{12}} & \ldots & K_{a_{12}} \\
K_{a_{12}} & K_{a_{11}} & \ldots & K_{a_{12}} \\
\vdots & \vdots & \ddots & \vdots \\
K_{a_{12}} & \ldots & \ldots & K_{a_{11}}
\end{array}\right)
$$

For more details refer to [21]. In the next session the solution of the centralized LQR problem is modified to a distributed control scheme.

\section{B. Distributed LQR Controller for Dynamically Decoupled Systems}

The collective dynamics of $N_{d}$ identical and decoupled dynamical subsystems can be described as:

$$
\dot{\tilde{\boldsymbol{x}}}(t)=\tilde{A} \tilde{\boldsymbol{x}}+\tilde{B} \tilde{\boldsymbol{u}}, \quad \tilde{\boldsymbol{x}}(0)=\tilde{\boldsymbol{x}}_{0}
$$

where $\tilde{\boldsymbol{x}}(t)$ and $\tilde{\boldsymbol{u}}(t)$ are the vectors which collect the states and inputs of the $N_{d}$ systems, while $\tilde{A}=I_{N_{d}} \otimes A$ and $\tilde{B}=$ $I_{N_{d}} \otimes B$, where $A$ and $B$ are defined as in (1). Systems (2) and (9) differ only in the number of subsystems.

The distributed optimal control problem is defined in [21], but in general its computation is considered as NP-hard problem. Therefore, the procedure for designing a suboptimal distributed controller is proposed in the following theorem.

Theorem 1. [21] Consider the (large-scale) LQR problem in (9), with cost function:

$$
J\left(\tilde{\boldsymbol{u}}(t), \tilde{\boldsymbol{x}}_{0}\right)=\int_{0}^{\infty}\left(\tilde{\boldsymbol{x}}(t)^{T} \tilde{Q} \tilde{\boldsymbol{x}}(t)+\tilde{\boldsymbol{u}}(t)^{T} \tilde{R} \tilde{\boldsymbol{u}}(t)\right) \mathrm{d} t
$$

where $\tilde{Q}$ is structured as in (4) with $\tilde{Q}_{i i}=\tilde{Q}_{1}$ for all $i=$ $1, \ldots, N$ and $\tilde{Q}_{i j}=\tilde{Q}_{2}$ for all $j=1, \ldots, N, i \neq j$.

Let $P_{\min }$ be the symmetric positive definite solution of the ARE associated with the centralized LQR problem, but of different size, i.e. the number of subsystems now is $N_{\min }=$ $d_{\max }(\mathcal{G})+1$. Then, $P_{\min }$ is of the following structure:

$$
P_{\text {min }}=\left(\begin{array}{cccc}
P_{11} & P_{12} & \ldots & P_{12} \\
P_{12} & P_{11} & \ldots & P_{12} \\
\vdots & \vdots & \ddots & \vdots \\
P_{12} & \ldots & \ldots & P_{11}
\end{array}\right)
$$

Furthermore, $P_{11}=P-\left(N_{\min }-1\right) P_{12}$, where $P$ is the symmetric positive definite solution to the single agent $L Q R$ problem in (7). Then, the distributed controller can be constructed as:

$$
\tilde{K}=I_{N_{d}} \otimes R^{-1} B^{T} P-M \otimes R^{-1} B^{T} P_{12}
$$

corresponding to the closed loop system:

$$
\tilde{A_{c l}}=\tilde{A}-\tilde{B} \tilde{K}=I_{N_{d}} \otimes A+\left(I_{N_{d}} \otimes B\right) \tilde{K}
$$

which is asymptotically stable. Matrix $M$ reflects the structure of the graph $\mathcal{G}$ and is given by $M=a I_{N_{d}}-b \boldsymbol{A}(\mathcal{G}), b \geq 0$, where $\boldsymbol{A}(\mathcal{G})$ is the adjacency matrix. Also, $a$ and $b$ have to satisfy $a-b d_{\max } \geq 0$ which follows from the gain margin properties of the proposed design.

Proof. See [21].

Remark 1.1. Theorem 1 implies that only one local controller can be used to control a collection of identical dynamically decoupled systems. Also, it is enough to solve a low-dimensional LQR problem from where the full-size distributed controller can be constructed.

In order to calculate the performance cost of the proposed distributed controller, the solution to Lyapunov equation associated with the problem has to be found; this is summarized in the next proposition.

Proposition 1. Consider the distributed controller designed as in Theorem 1 with the asymptotically stable closed loop system (13). The minimum cost is given by

$$
J\left(\tilde{\boldsymbol{u}}, \tilde{\boldsymbol{x}}_{0}\right)=\tilde{\boldsymbol{x}}_{0}^{T} \tilde{P} \tilde{\boldsymbol{x}}_{0}
$$

where $\tilde{P}$ is the unique solution of the following Lyapunov equation:

$$
\tilde{A}_{c l}^{T} \tilde{P}+\tilde{P} \tilde{A}_{c l}+\tilde{Q}+\tilde{K}^{T} \tilde{R} \tilde{K}=0 .
$$

Next, the solutions of the problems described in Section III-A and Section III-B are related using perturbation analysis.

\section{Perturbation Analysis}

Consider the distributed optimal control problem described in Section III-B with gain matrix

$$
\tilde{K}=I_{N_{d}} \otimes R^{-1} B^{T} P-M \otimes R^{-1} B^{T} P_{a_{12}} .
$$

In the case when the communication network is described by a complete graph $d_{\max }=N-1$. Therefore, there will exist an equivalence between (16) and the centralized large-scale gain matrix in (8). Edge(s) elimination from a fully connected network can be considered as a structured perturbation on the 
centralized large-scale problem. This is equivalent to a distributed communication network, where at least one subsystem is not connected to the remaining subsystems. Elimination of the $(i, j)$ th edge results in $\mathbf{A}(i, j)=\mathbf{A}(j, i)=0$ and also cancels the corresponding blocks in $K_{a}$. The resulting $K_{a}$ matrix will be called the perturbed gain matrix, $\tilde{K}$. Define $\Delta K=K_{a}-\tilde{K}$, e.g. in the case of $(1,2) \notin \mathcal{E}$,

$$
\Delta K=\left(\begin{array}{cccc}
0 & -R^{-1} B^{T} P_{a_{12}} & \ldots & 0 \\
-R^{-1} B^{T} P_{a_{12}} & 0 & \ldots & 0 \\
\vdots & \vdots & \ddots & \vdots \\
0 & \ldots & \ldots & 0
\end{array}\right) .
$$

The following result can now be established:

Theorem 2. Suppose that the assumptions of Theorem 1 hold and let $E=\tilde{P}-P_{a}$ where $P_{a}$ is defined in (5) and $\tilde{P}$ is the solution of the Lyapunov equation:

$$
\left(A_{a}-B_{a} \tilde{K}\right)^{T} \tilde{P}+\tilde{P}\left(A_{a}-B_{a} \tilde{K}\right)+\tilde{K}^{T} R_{a} \tilde{K}+Q_{a}=0
$$

Then, $E=E^{T}$ is the unique positive semi-definite solution of the following Lyapunov equation:

$$
\tilde{A}_{c l}^{T} E+E \tilde{A}_{c l}+(\Delta K)^{T} R_{a} \Delta K=0
$$

in which $\tilde{A}_{c l}=A_{a}-B_{a} R_{a}^{-1} B_{a}^{T} P_{a}+B_{a} \Delta K$ is Hurwitz. In particular, $E=E^{T}>0$ if and only if the pair $\left(A_{a}-B_{a} R_{a}^{-1} B_{a}^{T} P_{a}, \Delta K\right)$ is observable.

Proof. First note that since $A_{a}-B_{a} K_{a}$ and $A_{a}-B_{a} \tilde{K}$ are Hurwitz $P_{a} \geq 0$ and $\tilde{P} \geq 0$. Subtracting equation (5) from equation (18) shows (after some algebra) that $E$ is the solution of (19). Theorem 1 implies that $\tilde{A}_{c l}$ is Hurwitz which in turn implies that $E=E^{T} \geq 0$. Standard theory of Lyapunov equations now implies that $E$ is positive definite if and only if the pair $\left(\tilde{A}_{c l}, \Delta K\right)$ is observable, which is equivalent to the observability of the pair $\left(A_{a}-B_{a} R_{a}^{-1} B_{a}^{T} P_{a}, \Delta K\right)$.

\section{MAin RESUlTS}

Using Theorem 2 we can draw some conclusions on the cost increase that is imposed by distributed design. Not surprisingly we have the following result:

Proposition 2. The cost imposed by the distributed LQR problem will be always equal or higher than the cost imposed by the centralized design.

Proof. Although the result is obvious (the cost of the centralized optimal controller cannot exceed the cost of the distributed controller) we give a direct proof. The result is immediate if the distributed controller is not stabilizing, so assume that the distributed cost is finite. From Theorem 2 it is known that $\tilde{P}=P_{a}+E$ with all matrices being symmetric positive semi-definite. Applying Weyl's inequality (see [22]) to $\tilde{P}=P_{a}+E$ gives:

$$
\lambda_{k}\left(P_{a}\right)+\lambda_{n}(E) \leq \lambda_{k}(\tilde{P}) \text { for any } 1 \leq k \leq n
$$

where the eigenvalues are indexed in decreasing order. The required result follows since $\lambda_{n}(E) \geq 0$.
A natural question arising from Proposition 2 is under what conditions, if any, the two costs are equal. The following result gives necessary and sufficient conditions for equality of the two costs:

Theorem 3. The cost of a stabilizing distributed controller defined in Theorem 1 is equal to the cost of the centralized optimal LQR controller if and only if the pair $\left(A_{a}-\right.$ $\left.B_{a} R_{a}^{-1} B_{a}^{T} P_{a}, \Delta K\right)$ is unobservable and $\tilde{\boldsymbol{x}}_{0} \in \operatorname{Ker}(E)$ where $E=\tilde{P}-P_{a}$.

Proof. The cost of a stabilizing distributed controller is:

$$
J\left(\tilde{\boldsymbol{u}}, \tilde{\boldsymbol{x}}_{0}\right)=\tilde{\boldsymbol{x}}_{0}^{T} \tilde{P} \tilde{\boldsymbol{x}}_{0}=\tilde{\boldsymbol{x}}_{0}^{T} P_{a} \tilde{\boldsymbol{x}}_{0}+\tilde{\boldsymbol{x}}_{0}^{T} E \tilde{\boldsymbol{x}}_{0}
$$

in which the first term on the right-hand-side of the last equality represents the optimal LQR cost of the centralized controller. Since $E=E^{T} \geq 0$, the term $\tilde{\boldsymbol{x}}_{0}^{T} E \tilde{\boldsymbol{x}}_{0}$ is zero if and only if $E$ is singular and $\tilde{\boldsymbol{x}}_{0} \in \operatorname{Ker}(E)$, the first condition being equivalent to the unobservability of the pair $\left(A_{a}-B_{a} R_{a}^{-1} B_{a}^{T} P_{a}, \Delta K\right)$.

It follows that if we can identify cases for which $E$ is singular, then optimality is preserved. Results are presented in Theorem 5, but before stating and proving this theorem we need the following preliminary result which characterises the spectrum of the (large-scale) closed-loop centralized LQR system:

Theorem 4. Let $A_{c l}=A-B R^{-1} B^{T} P$ be the closed-loop matrix of the (single system) LQR problem for the system in (1) with state and control weighting matrices $Q_{1}$ and $R$, respectively, and where $P$ is the symmetric positive definite solution of (7). Also, let $A_{c l_{a}}=A_{a}-B_{a} R_{a}^{-1} B_{a}^{T} P_{a}$ be the closed-loop matrix of the (large-scale) centralized $L Q R$ problem in (2) with state and control weighting matrices $Q_{a}$ and $R_{a}$ respectively, where $P_{a}$ is the symmetric positive definite solution of (5). Assume that $P_{a}$ is decomposed into $N^{2}$ blocks of dimension $n \times n$ as in (6). Then, the spectrum of $A_{c l_{a}}$, i.e. $\mathcal{S}\left(A_{c l_{a}}\right)$ is given by:

$$
\mathcal{S}\left(A_{c l_{a}}\right)=\mathcal{S}\left(A_{c l}\right) \cup \underbrace{\mathcal{S}\left(A_{c l_{1-2}}\right) \cup \ldots \cup \mathcal{S}\left(A_{c l_{1-2}}\right)}_{(N-1) \text { times }}
$$

where $A_{c l_{1-2}}=A-B R^{-1} B^{T}\left(P_{a_{11}}-P_{a_{12}}\right)$, in which $P_{a_{11}}$ and $P_{a_{12}}$ are $n \times n$ blocks of $P_{a}$ defined in (6).

Proof. By substituting $P_{a}$ in (6) into $A_{c l_{a}}$ we get:

$$
A_{c l_{a}}=\left(\begin{array}{cccc}
A-X P_{a_{11}} & -X P_{a_{12}} & \ldots & -X P_{a_{12}} \\
-X P_{a_{12}} & A-X P_{a_{11}} & \ldots & -X P_{a_{12}} \\
\vdots & \vdots & \ddots & \vdots \\
-X P_{a_{12}} & \ldots & \ldots & A-X P_{a_{11}}
\end{array}\right) .
$$

$A c l_{a}$ can be transformed into a block lower-triangular matrix through the similarity transformation $A_{c l_{t}}=T A_{c l_{a}} T^{-1}$, where the transformation matrix $T$ is given by

$$
T=\left(\begin{array}{ccccc}
I & -I & 0 & \ldots & 0 \\
0 & I & -I & \ldots & 0 \\
\vdots & \vdots & \ddots & \ddots & \vdots \\
0 & 0 & \ldots & \ddots & -I \\
0 & 0 & \ldots & \ldots & I
\end{array}\right) .
$$


Therefore, $A c l_{t}$, becomes:

$$
A_{c l_{t}}=\left(\begin{array}{cccc}
A-X P_{a_{1-2}} & \cdots & 0 & 0 \\
\vdots & \ddots & \vdots & \vdots \\
0 & \cdots & A-X P_{a_{1}-2} & 0 \\
-X P_{a_{12}} & \cdots & -(N-1) X P_{a_{12}} & A-X P
\end{array}\right)
$$

where $P_{a_{1-2}}=P_{a_{11}}-P_{a_{12}}$ and $P=P_{a_{11}}+(N-1) P_{a_{12}}$ is the symmetric positive definite solution to a single system $L Q R$ problem. Since eigenvalues of a matrix are preserved under similarity transformations equation (21) follows.

We can now prove the following:

Theorem 5. Suppose that a single link is removed from a complete network with at least four subsystems $(N \geq 4)$. Then $E$ is singular.

Proof. The assumption $N \geq 4$ ensures that the assumptions of Theorem 1 are satisfied. Thus $\tilde{A}_{c l}$ is Hurwitz and $E=E^{T} \geq$ 0 . To show that $E$ is singular it suffices to show that the pair $\left(A_{a}-B_{a} R_{a}^{-1} B_{a}^{T} P_{a}, \Delta K\right)$ is unobservable which is equivalent to the existence of $\lambda \in \mathbb{C}$ such that the matrix $\left(\begin{array}{c}A_{c l_{a}}-\lambda I \\ \Delta \bar{K}\end{array}\right)$ is rank deficient. For the network of $N$ agents and link removal between agents 1 and 2 this matrix can be written as:

$$
\left(\begin{array}{cccc}
A_{11}-\lambda I & -X P_{a_{12}} & \ldots & -X P_{a_{12}} \\
-X P_{a_{12}} & A_{11}-\lambda I & \ldots & -X P_{a_{12}} \\
\vdots & \vdots & \ddots & \vdots \\
-X P_{a_{12}} & -X P_{a_{12}} & \cdots & A_{11}-\lambda I \\
-0^{-} & -\bar{R}^{-\mathrm{T}} \bar{B}^{T} \bar{P}_{a_{12}} & \cdots & 0 \\
-R^{-1} B^{T} P_{a_{12}} & 0 & \cdots & 0 \\
\vdots & \vdots & \ddots & \vdots \\
0 & 0 & \ldots & 0
\end{array}\right)
$$

where $A_{11}=A-X P_{a_{11}}$. Next we introduce the state-space transformation $\left(\begin{array}{c}T\left(A_{c l_{a}}-\lambda I\right) T^{-1} \\ -\bar{K} \overline{T^{-1}}\end{array}\right)$ in which $T$ is chosen as:

$$
T=\left(\begin{array}{c:c}
T_{11} & T_{12} \\
\hdashline \bar{O}^{+} & \bar{I}^{-}
\end{array}\right)
$$

where $O \in \mathbb{R}^{(N-4) n \times 4 n}$ and $I \in \mathbb{R}^{(N-4) n \times(N-4) n}$ denote the zero and the unit matrix, respectively, and

$$
\begin{aligned}
T_{11} & =\left(\begin{array}{cccc}
I & I & I & I \\
0 & I & 0 & 0 \\
0 & -I & I & 0 \\
0 & I & I & I
\end{array}\right) \in \mathbb{R}^{4 n \times 4 n}, \\
T_{12} & =\left(\begin{array}{c}
I \\
0 \\
\vdots \\
0 \\
I
\end{array}\right)\left(\begin{array}{lll}
I & \ldots & I
\end{array}\right) \in \mathbb{R}^{4 n \times(N-4) n} .
\end{aligned}
$$

It can be seen, after some algebra, that under $T$ the transformed matrix in (24) loses rank along the third column if $\lambda$ is chosen as an eigenvalue of the matrix $A-X\left(P_{a 11}-P_{a 12}\right)$ (using Theorem 4). Since the rank of a matrix remains invariant under similarity transformations the system $\left(\tilde{A}_{c l}, \Delta K\right)$ is unobservable in this case and hence
$E$ is singular. The result can also be generalized if an arbitrary (rather than link $(1,2)$ ) is removed (see subsequent remark).

Remark 5.1. The fact that $\Delta K$ can have a different structure, depending on the choice of an edge that is eliminated, will not change the eigenvalue distribution in $\tilde{P}$, nor in $\tilde{A}_{c l}$. Therefore, same conclusions can be drawn for any of $\frac{N(N-1)}{2}$ configurations corresponding to a single-link removal from the complete network of $N$ agents.

Remark 5.2. The performance loss analysis can be extended along various directions. A natural question arising from Proposition 2 and Theorem 3 is to identify near-optimal distributed configurations for which the cost increase from the optimal level will be minimal. This corresponds to the term $\tilde{\boldsymbol{x}}_{0}^{T} E \tilde{\boldsymbol{x}}_{0} \geq \epsilon\left\|\tilde{\boldsymbol{x}}_{0}\right\|^{2}$ with $\lambda_{\min }(E)=\epsilon>0$. Similarly, $\lambda_{\max }(E)$ is a measure of the maximal deviation from optimality if $\tilde{\boldsymbol{x}}_{0}$ is aligned in the direction of the corresponding eigenvector. A final measure of performance loss is the average cost increase over all initial state directions which can be described as $\frac{\text { trace }(E)}{n N_{d}}$. It is assumed that the initial state vector is uniformly distributed on the surface of an n-dimensional hyper-sphere, $\left\|\tilde{\boldsymbol{x}}_{0}\right\|=1$.

The complexity of analysis increases with the number of subsystems, and the number of eliminated edges, but same conclusions would apply. Proofs are omitted due to space restrictions, but an numerical example is given next.

Example: Consider a network of $N=6$ identical, dynamically decoupled agents represented by a complete graph. Their collective dynamics is given by

$$
\dot{\tilde{\boldsymbol{x}}}(t)=\tilde{A} \tilde{\boldsymbol{x}}+\tilde{B} \tilde{\boldsymbol{u}}, \quad \tilde{\boldsymbol{x}}(0)=\tilde{\boldsymbol{x}}_{0}
$$

where $\tilde{A}=I_{6} \otimes A$ and $\tilde{B}=I_{6} \otimes B$ with $A$ and $B$ defined as

$$
A=\left[\begin{array}{rrr}
-1 & 0 & -2 \\
-2 & -3 & -4 \\
1 & 0 & -1
\end{array}\right], B=\left[\begin{array}{rr}
1 & 1 \\
0 & 2 \\
-1 & 3
\end{array}\right] \text {. }
$$

The cost function defined in (3) uses the following weights for the state information: $Q_{a_{i i}}=Q_{1}=I_{3}$ and $Q_{a_{i j}}=Q_{2}=$ $I_{3}$, while the weight on the control effort is $R_{a}=I_{6} \otimes R$ with $R=I_{2}$. For a given initial state vector $\tilde{\boldsymbol{x}}_{0}$, such that $\left\|\tilde{\boldsymbol{x}}_{0}\right\|=1$, we get the stabilizing centralized LQR solution, $P_{a}$ and therefore the optimal (minimal) cost. For this case the cost measures are given in Table I.

TABLE I

COST MEASURES FOR OPTIMAL (CENTRALIZED) LQR DESIGN

\begin{tabular}{|c|c|c|}
\hline Minimum Cost & Average Cost & Maximum Cost \\
\hline 0.1027 & 1.3426 & 2.6006 \\
\hline
\end{tabular}

Next, we consider a number of different distributed configurations obtained by removing links from a complete network. The performance loss is measured by using alternative methods presented in Remark 5.2 and results are given in Table II. 
TABLE II

COST MEASURES FOR DISTRIBUTED CONFIGURATIONS

\begin{tabular}{|l|c|c|c|}
\hline & $\begin{array}{c}\text { Single cut } \\
\left(d_{\max }, d_{\min }\right)\end{array}$ & $\begin{array}{c}\text { Double cut } \\
\left(d_{\max }, d_{\min }\right)\end{array}$ & $\begin{array}{c}\text { Triple cut } \\
\left(d_{\max }, d_{\min }\right)\end{array}$ \\
\hline \hline Minimum & $0.1031(5,4)$ & $0.1035(5,4)$ & $0.1027(4,4)$ \\
Cost & & $0.1039(5,3)$ & $0.1043(5,3)$ \\
\hline & & & $0.1050(5,2)$ \\
Average & $1.3449(5,4)$ & $1.3471(5,4)$ & $1.3538(4,4)$ \\
Cost & & $1.3474(5,3)$ & $1.3496(5,3)$ \\
& & & $1.3503(5,2)$ \\
Maximum & $2.6088(5,4)$ & $2.6088(5,4)$ & $2.6300(4,4)$ \\
Cost & & $2.6178(5,3)$ & $2.6178(5,3)$ \\
& & & $2.6270(5,2)$ \\
\hline
\end{tabular}

As an additional information, the maximum and minimum degree are given for each configuration.

It can be seen that all costs are highly dependent on how well network is connected (i.e. they all increase as the minimum degree, $d_{\min }$, drops for a fixed $d_{\max }$ ). Additionally, for each configuration there is at least one direction for which optimality is preserved compared to the centralized design. For example in the case of triple cut with $d_{\min }=2 E$ is singular $(\operatorname{rank}(E)=9)$ which implies the equality in costs for specific directions as claimed in Theorem 3 and Theorem 5. Note that this does not necessarily imply that this direction coincides with the direction of the eigenvector corresponding to $\lambda_{\min }\left(P_{a}\right)$. Detailed results are omitted due to the space restrictions. Note that when three links are removed in the case under consideration, equality with $\lambda_{\min }\left(P_{a}\right)$ occurs only for a configuration corresponding to a minimum and maximum degree equal 4 . Therefore, the minimum value among all minimal costs occurs when all agents share the same degree and we have so called regular network. The relation between LQR cost and the connectivity properties of the network will be fully investigated in future work.

\section{CONCLUSIONS}

The paper has considered a distributed LQR design framework for a network of dynamically decoupled multi-agent systems with identical dynamics. Necessary and sufficient conditions have been derived for which a distributed control configuration pattern arising from the optimal centralizing solution does not entail loss of performance. It was shown that these conditions are always satisfied for systems with communication/control networks corresponding to complete graphs with a single link removed. The results are useful for quantifying performance loss due to decentralization and for designing optimal or near-optimal distributed control schemes. Future work will attempt to establish precise correlations between performance cost and the connectivity properties of the network.

\section{REFERENCES}

[1] A. Jadbabaie, J. Lin, and A. S. Morse, Coordination of groups of mobile autonomous agents using nearest neighbor rules, IEEE Trans. Automatic Control, vol. 48, no. 6, pp. 988-1001, 2003.
[2] J. A. Fax and R. M. Murray, Information flow and cooperative control of vehicle formations, IEEE Trans. Automatic Control, vol. 49, no. 9, pp. 1465-1476, 2004.

[3] Y. Chen and Z. Wang, Formation control: a review and a new consideration, in Proc. the 2005 IEEE/RSJ International Conference on Intelligent Robots and Systems, pp. 3181-3186, 2005.

[4] R. W. Beard, J. Lawton, and F. Y. Hadaegh, A coordination architecture for spacecraft formation control, IEEE Trans. Contr. Sys. Tech., vol. 9 , no. 6, pp. 777-790, 2001.

[5] R. Olfati-Saber, Flocking for multi-agent dynamic systems: Algorithm and theory, IEEE Trans. Automatic Control, vol. 51, no. 3, pp. 401-420, 2006.

[6] G. Tanner, A. Jadbabaie, and G. J. Pappas, Flocking in fixed and switching networks, IEEE Trans. Automatic Control, vol. 52, no. 5, pp. 863-868, 2007.

[7] J. Cortes, S. Martinez, T. Karatas, and F. Bullo, Coverage control for mobile sensing networks, IEEE Trans. Robotics and Automation, vol. 20, no. 2, pp. 243-255, 2004.

[8] C. Tomlin, G. J. Pappas, and G. J. Sastry, Conflict resolution for air traffic management: A study in multi-agent hybrid systems, IEEE Trans. Automatic Control, vol. 43, no. 4, pp. 509-521, 1998.

[9] F. Paganini, J. Doyle, and S. Low, Scalable laws for stable network congestion control, in Proc. 40th IEEE Conf. Decision and Control, pp. 185-190, 2001.

[10] P. Massioni and M. Verhagen, Distributed control for identical dynamically coupled systems: A decomposition approach, IEEE Trans. Automatic Control, vol. 54, no. 1, pp. 124-135, 2009.

[11] B. Bamieh, F. Paganini, and M. A. Dahleh, Distributed control of spatially invariant systems, IEEE Trans. Automatic Control, vol. 47, no. 7, pp. 1091-1107, 2002.

[12] C. Langbort, R. S. Chandra, and R. D'Andrea, Distributed control design for systems interconnected over an arbitrary graph, IEEE Trans. Automatic Control, vol. 49, no. 9, pp. 1502- 1519, 2004

[13] Z. Lin, B. Francis, and M. Maggiore, State agreement for continuous time coupled nonlinear systems, SIAM Journal of Control Optim., vol. 46, pp. 288-307, 2007.

[14] A. P. Popov and H. Werner, A robust control approach to formation control, in Proc. 10th European Control Conf., Budapest, Hungary, pp. 4428-4433, 2009.

[15] Y. C. Cao and W. Ren, Optimal linear-consensus algorithms: An LQR perspective. IEEE Trans. on Systems, Man, and Cybernetics-Part B: Cybernetics, vol. 40, no. 3, pp. 819-830, 2010.

[16] C. Langbort, and V. Gupta, Minimal interconnection topology in distributed control design, SIAM J. Control Optim., vol. 48, no. 1, pp. 397413, 2009.

[17] P. Deshpande, P. Menon, C. Edwards, and I. Postlethwaite, A distributed control law with guaranteed LQR cost for identical dynamically coupled linear systems, in Proc. American Control Conference, pp. 5342-5347, 2011.

[18] Q. Wang, C. Yu, H. Gao, and F. Liu, A distributed control law with guaranteed convergence rate for identically coupled linear systems, in Proc. 12th European Control Conf., Zurich, Switzerland, pp. 2286-2291, 2013.

[19] F. Lin, M. Fardad, and M. R. Jovanovic, Augmented Lagrangian approach to design of structured optimal state feedback gains, IEEE Trans. on Automatic Control, vol. 56, no. 12, pp. 2923-2929, 2011.

[20] F. Lin, M. Fardad, and M. R. Jovanovic, Optimal control of vehicular formations with nearest neighbor interactions, IEEE Trans. on Automatic Control, vol. 57, no. 9, pp. 2203-2218, 2012.

[21] F. Borrelli and T. Keviczky, Distributed LQR design for identical dynamically decoupled systems, IEEE Trans. Automatic Control, vol. 53, no. 8, pp. 1901-1912, 2008.

[22] R. A. Horn and C. R. Johnson,, Topics in Matrix Analysis, Cambridge University Press, 1994 